\title{
Constitutive Developmental Modeling of Genomic Integrity and Epigenetic Interactivity in Neuroblastoma
}

\begin{abstract}
The essential formulas of possible progression or non-progression are dimensionally and potentially highly divergent systems of operative aggressive growth and spread of a neuroblastoma. As such, an essential integrity of a given genomic profile and the interactivities of epigenetic factors reconstitute in clinical and pathologic terms the emergence of tumor evolution that proposes highly divergent profiles for possible resolution of the lesion, but also as biologic substrate for developmental emergence of malignant change and of progression of tumor growth and spread. The performance profile is historic characterization of a neoplasm that may regress completely in terms of such interaction between integral genome and epigenetic modeling of dimensional nature. The projected attributes of a neuroblastoma lesion is perforce dimensional reconstitution of such developmental dynamics as best characterized by systems of parametrically driven reconstitution of the genome of the neoplastic cells.
\end{abstract}

\section{Introduction}

Neuroblastoma, as a specific neoplastic type of involvement of the post ganglionic sympathetic nervous system, recapitulates dimensions of progression that define a high degree of variability in clinical outcome in the individual pediatric patient. Occasionally, intratumoral heterogeneity shows the co-existence of MYCN amplified and non-MYCN amplified tumor cell clones [1]. The considerable clinical variability is contrasted to the highly penetrant evolutionary history of inherited versus somatic mutability of the cell genome affected. Novel tumorigenic miRNA gene networks are found and miRNA 204 as a tumor suppressor regulates MYCN expression in neuroblastoma oncogenesis by inhibiting a sub network of oncogenes associated with MYCN-amplified neuroblastoma and poor prognosis [2]. It is further to such setting that the Anaplastic Lymphoma Kinase (ALK) constitutes a frequent germ line mutability that provokes dimensions for a multi-stage driven genomic evolution in its own right.

The proportionality of amplification of the MYCN is also a variable index for malignant transformation dependent on genomic evolution and thus is considered a valid target for therapeutic intervention. Conceptually, genomic evolution as a fully independent variable parameter includes MYCN in terms of substantial driving formula for a neuroblastoma lesion that often regresses but also a tumor type that can in several patients behave aggressively and metastasize widely.

\section{Neuroblastoma variability}

This variability of neuroblastoma is intriguing within the confined extension of both hereditary and somatically acquired tumor subsets that evolve in terms of a historic dependency for

\section{Journal of}

\section{Cancer Sciences}

\author{
Lawrence M. Agius* \\ Department of Pathology, University of Malta Medical School, Europe \\ *Address for Correspondence \\ Lawrence M. Agius, Guzeppe caruana Street, Europe, Tel: 356- \\ 21451752; E-mail : lawrence.agius@um.edu.mt \\ Submission: 22 May 2018 \\ Accepted: 15 June 2018 \\ Published: 22 June 2018 \\ Copyright: () 2018 Agius LM. This is an open access article distributed \\ under the Creative Commons Attribution License, which permits \\ unrestricted use, distribution, and reproduction in any medium, provided \\ the original work is properly cited.
}

variability in genomic evolution particularly in terms of clinical progression within the given individual patient with neuroblastoma. A pair of sense/antisense long noncoding RNA (6p22lncRNAs) encoded by CASC15 and NBAT1 located at the neuroblastoma risk associated 6p22.3 locus are tutor suppressors and show reduced expression in high-risk neuroblastoma [3]. In such terms, genomic variability is a product phenomenon in its own right in terms that are pronounced in clinical outcome statistics. Somatic chromosomal copy number aberrations are associated with patient outcome, particularly in low and intermediate risk neuroblastoma patients and together with distal 6q loss constitute novel markers for poor survival in high risk patients with neuroblastoma [4]. The further concept of driven machinery formulation is clinically a series of indications for a highly plastic evolutionary attribute that constitutes the parametric support and prognostically validated dimensions for further growth in spread and tumor cell proliferation in children with the aggressive form of neuroblastoma. Particularly intriguing is the phenomenon of regression of a common tumor type that bears significant import to cancer multi-stage attributes.

\section{Genome integrity}

There thus emerges machinery in which genomic integral dimensions constitute a series of sometime susceptibility in progression of the genome itself. In such terms, especially with regard to possible spontaneous tumor regression, the genome not only constitutes a series of vulnerabilities to malignant transformation but also an essential integrity for resolution of such malignant change. The molecular basis for aggressive neuroblastoma remains elusive which shows a paucity of recurrent somatic mutations at diagnosis; a 10 protein transcriptional module entered around a TEAD5-MYCN positive feedback loop emerged as the regulatory driver of high risk subtype associated with MYCN amplification [5]. Neuroblastoma is constitutive formulation of driven carcinogenesis that is fully dependent on genomic integral evolutionary change as tumors present clinically and which also suffer from a host a widely divergent attributes for vulnerability for possible different clinical outcome.

D-Limonene inhibits carcinogenesis and promotes tumor regression as it modulates autophagic markers that are linked to cancer and neurodegeneration [6].

Such dimensional variability is a heterogeneous susceptibility of the genomic biology as integral expression in terms beyond the 
clinical emergence of the tumor type of neuroblastoma and as further formulated in specific terms of clinical progression.

\section{System profiles}

The benign form of neuroblastoma is attribute dimension for a series of ongoing system profiles that parametrically drive or do not drive the outcome dynamics of pathologic and clinical aggressiveness of a given neuroblastoma lesion. Perforce dimensions in the evolutionary dynamics of an integral genomic biology in a given global group of neuroblastoma lesions clearly calls into operative dimensions the dysregulation of a malignancy type phenomenon that includes possible clinical regression as a constitutive attribute of the genomic biology in the global neuroblastic lesion.

Gene copy number alterations are the hallmark of both murine and human disease and frequently affect syntenic genomic regions; despite low mutational load, the genes mutated in murine disease are enriched for genes mutated in human disease [7].

Parametric oncogenesis thus emerges as referential dynamics of attributes of an integral genome within the global dimensions of a given neuroblastoma neoplasm as also well projected in terms of the constitutive progression or resolution of the lesion.

Pan cancer analyses that examine commonalities and differences among various cancer types is an effective way to obtain novel insights into cancer biology; small mutation and structural/copy-number variants (that correlate with germline variants) exist [8].

\section{Genome evolution}

Such proposed inherent nature of genome evolution is well exemplified dynamics for a multi-stage oncogenesis that can potentially prove reversible in terms of integral genomic driving formulas of the lesion. Cancer appears a complex evolutionary system as validated by in depth molecular analyses of tumor progression; intrinsic and acquired plasticity of cancer cells allow for evasion of growth factor limitations, apoptotic signals or attacks from the immune system [9]. It is perhaps significant to consider the proportionality of mutations and amplifications that are based on dual interactions between various nucleotide polymorphisms on the one hand and also especially of epigenetic factors that affect the vulnerabilities of a given genome type that characterizes the individual stages of progression of a neuroblastoma lesion.

Germ line variability in dimensional reconstitution is hereby constitute image formula in the development of neural crest derivatives and as such is a non somatically acquired attribute per se. Regression of neuroblastoma constitutes a highly characterized dimension within systems of a highly influential epigenesis based in turn on integral dynamics of a given genome that globally drives the individual neuroblastoma cell in terms of a global tumor lesion [10].

\section{Dual settings}

This neoplasm shows a particularly wide spectrum of possible clinical outcome and its prognosis is determined by numerous biologic and genetic factors; investigation allows stratification in pretreatment risk groups but it has become clear that genetic patterns are more complex than previously conceived [11]. Hence, there emerges a dual setting of a given genome integrity and of epigenesis based presumably on circulating cytokines encompassed by integral genomic dynamics. In such terms, operative factors in the evolution of genome and epigenesis clearly constitute a highly plastic dynamics within the given development of a neuroblastoma lesion. Such a phenomenon is constitutive machinery in terms of ongoing evolution of an integral genome per se and as further dictated by dimensions of a given stage in progression of a neuroblastoma that is highly heterogeneous both biologically and pathologically. Amplification of the oncogenic transcription factor MYCN is a defining feature of high-risk neuroblastoma with highly tumor specific MYC target gene signatures [12]. Such dimensions clearly denote the constitutively driven formulas for interactions between integral and dynamic reconstitution of injury to genome and epigenetic response.

\section{Concluding Remarks}

Performance dynamics are significant models of influence and interactivity that dimensionally characterize a potentially large number of operative factors as constituted by both integral genomic evolution and epigenetic dynamics [13]. A realistic projection for dynamics of a given neuroblastoma lesion is character dimension in terms of a phenomenon that includes the modeled formulas for interaction as dynamics per se evolve within the individual patient. Developmental considerations in especially the younger pediatric patient group allows for characterization that is projected as dual pathways for possible re-constitution of both the genome and the epigenetic machinery in terms that dimensionalize dynamics of tumor progression or non-progression.

\section{References}

1. Berbegall AP, Bogen D, Putschger U, Beiska K, Bown N, et al. (2018) Heterogeneous MYCN amplification in neuroblastoma: a SIOP Europe neuroblastoma study. $\mathrm{Br} \mathrm{J}$ Cancer.

2. Ooi CY, Carter DR, Liu B, Mayoh C, Beckers A, et al. (2018) Network modeling of microRNA-mRNA interactions in neuroblastoma tumorigenesis identifies miR-204 as a direct inhibitor of MYCN. Cancer Res.

3. Mondal T, Juvvuna PK, Kirkeby A, Mitra S, Kosalai ST, et al. (2018) Senseantisense IncRNA pair encoded by locus 6 p22.3 determines neuroblastoma susceptibility via the USP36-CHD7-SOX9 regulatory axis. Cancer Cell pp. 417-434.

4. Depuydt P, Boeva V, Hocking TD, Cannoodt R, Ambros IM, et al. (2018) Genomic amplifications and distal $6 q$ loss: novel markers for poor survival in high-risk neuroblastoma patients. J Natl Cancer Inst.

5. Rajbhandari P, Lopez G, Capdevila C, Salvatori B, Yu J, et al. (2018) Crosscohort analysis identifies a TEAD5-MYCN positive feedback loop as the core regulatory element of high-risk neuroblastoma. Cancer Discov 8: 582-599.

6. Berliocchi L, Chiappini C, Adornato A, Gentile D, Cerri S, et al. (2018) Early LC3 lipidation induced by d-limonene does not rely on mTOR inhibition, ERK activation and ROS production and it is associated with reduced clonogenic capacity of SH-SY5Y neuroblastoma cells. Phytomedicine 40: 98-105.

7. De Wilde B, Beckers A, Lindner S, Kristina A, De Preter K, et al. (2017) The mutational landscape of MYCN, Lin28b and ALK ${ }^{F 1174 L}$ driven murine neuroblastoma mimics human disease. Oncotarget 9: 8334-8349.

8. Grobner SN, Worst BC, Weischenfeldt J, Buchhalter I, Kleinheinz K, et al. (2018) The landscape of genomic alterations across childhood cancers. Nature 555: 321-327.

9. Schulte M, Koster J, Rahmann S, Schramm A (2018) Cancer evolution, mutations, and clonal selection in relapse neuroblastoma. Cell Tissue Res 372: $263-268$. 
Citation: Agius LM. Constitutive Developmental Modeling of Genomic Integrity and Epigenetic Interactivity in Neuroblastoma. J Cancer Sci. 2018;5(1): 3.

\section{ISSN: $2377-9292$}

10. Joshed JI, Dyberg C, Fransson S, Wickstrom M (2018) Molecular mechanisms and therapeutic targets in neuroblastoma. Pharmacol Res 131:164-176.

11. Costa RA, Seuanez HN (2018) Investigation of major genetic alterations in neuroblastoma. Mol Biol Rep 45: 287-295.
12. Zeid R, Lawlor MA, Poon E, Reyes JM, Fulciniti M, et al. (2018) Enhancer invasion shapes MYCN-dependent transcriptional amplification in neuroblastoma. Nat Genet 50: 515-523.

13. Nakagawara A, Li Y, Izumi H, Muramori K, Inada H, et al. (2018) Neuroblastoma. Jpn J Clin Oncol 58: 214-241. 